\title{
Estrategias de enseñanza basada en enfoque constructivista y evaluación de aprendizajes en Instituciones Educativas, Castilla-Piura
}

Teaching strategies based on a constructivist approach and assessment of learning in Educational Institutions, Castilla-Piura

\author{
a. Claudia Juliana Maza Sócola \\ claudiajulianamaza@gmail.com \\ Código ORCID: 0000-0001-6908-2196 \\ Universidad César Vallejo, Perú
}

\author{
| Artículo recibido en octubre 2020 \\ | Arbitrado en noviembre 2020 \\ | Publicado en enero 2021
}

\section{Resumen}

La presente investigación tuvo como objetivo determinar la relación que existe entre las estrategias de enseñanza basada en enfoque constructivista y la evaluación de aprendizajes en Instituciones Educativas de Castilla-Piura. El estudio se fundamentó en un enfoque cuantitativo de diseño no experimental, descriptivo correlacional con dos variables; una población-muestra de 88 docentes, en cuatro instituciones educativas. Para ello se diseñaron dos instrumentos, estructurados en preguntas con escala de Likert. Se validó por medio del juicio de expertos y la confiabilidad a través del índice Alfa de Cronbach. Los datos fueron procesados y analizados utilizando métodos estadísticos descriptivos e inferenciales; así como el coeficiente de correlación de Spearman. Luego de analizar e interpretar los resultados, se concluye que las estrategias de enseñanza se relacionan de manera significativa con la evaluación de aprendizajes; esto implica que una mejora en la evaluación de los aprendizajes requiere mejorar las estrategias de fase inicial.

\footnotetext{
Abstract

The present research aims to determine the relationship that exists between teaching strategies based on a constructivist approach and the assessment of learning in Educational Institutions of Castilla-Piura. The study is based on a quantitative approach of non-experimental, descriptive correlational design with two variables; a sample population of 88 teachers, in four educational institutions. For this, two instruments were designed, structured in questions with a Likert scale. It was validated through expert judgment and reliability through Cronbach's Alpha index. The data were processed and analyzed using descriptive and inferential statistical methods; as well as the Spearman Correlation coefficient. After analyzing and interpreting the results, it is concluded that the teaching strategies are significantly related to the assessment of learning; This implies that an improvement in the evaluation of learning requires improving the initial phase strategies.
}

\section{Palabras clave:}

Estrategias de enseñanza; estrategias de fase inicial; estrategias de fase intermedia; estrategias de fase terminal: evaluación

\section{Keywords:}

Teaching strategies; initial phase strategies; intermediate phase strategies; terminal phase strategies; learning assessment 


\section{INTRODUCCIÓN}

Actualmente el mundo se desenvuelve en contextos cambiantes, realidades difícilmente predecibles; los seres humanos del siglo XXI deberán enfrentar grandes brechas tecnológicas, cambio climático, problemáticas sociales cada vez más complejas, crear soluciones innovadoras, entre otros retos. Frente a esta realidad la educación en este siglo no se puede abordar desde estrategias dirigidas a conocimientos segmentados, evaluaciones de productos, sin tomar en cuenta los procesos de construcción de los aprendizajes. Es por ello que la presente investigación aborda la relación entre las estrategias de enseñanza basadas en un enfoque constructivista y la evaluación de los aprendizajes en Instituciones Educativas en Castilla-Piura. La investigación abordó el contexto global y particular del ámbito de estudio y permitió conocer la importancia de estas dos variables y de la necesidad de poseer una visión constructivista sobre las mismas.

El conocimiento es una construcción no una representación que hace el sujeto del objeto; por consiguiente, el conocimiento es producto de la interpretación del ser humano y no de la observación propiamente dicha. Durante este proceso de acercamiento a la realidad el criterio de validación de la realidad es la intersubjetividad; lo cual es un acuerdo provisional e inexacto entre los sujetos y requiere confrontación y triangulación. Estas ideas se oponen a la objetividad o exactitud formuladas por el positivismo, durante buena parte del siglo XX, donde se defendió el método científico como una posibilidad absoluta para estudiar la realidad.

En tal sentido, el constructivismo como enfoque psicológico propone entre sus fines, lograr tan solo una aproximación a la verdad, es decir, un conocimiento relativo, cambiante, discutible, holístico, en el cual el sujeto es participante, comprometido, ético. Para Ernst von Glasersfeld y Humberto Marturana (citados por Díaz-Barriga y Hernández, 2002) "el conocimiento se construye de manera subjetiva por lo que no es posible formar representaciones objetivas ni verdaderas de la realidad, lo que existe son formas viables o efectivas de actuar sobre la misma" (p.231). Desde esta perspectiva la construcción del conocimiento se origina de procesos de interacción de los sujetos con su entorno; es el sujeto el que le da significado a dichos procesos y construye sus aprendizajes a partir de ellos. En el contexto educativo, el alumno le da significado a la información que recibe; para lograrlo es fundamental el apoyo del entorno en el que se desenvuelve. Por ello resulta muy importante el diseño de estrategias de enseñanza basadas en el constructivismo para alcanzar aprendizajes significativos $\mathrm{y}$ pertinentes para el sujeto, donde la información nueva por aprender se relaciona con la información ya existente en la estructura del alumno.

Por otra parte, se debe destacar la función pedagógica de la evaluación y la importancia del proceso evaluativo; pues dichos procesos se centran en la calificación y no se ocupa de las causas y cómo se han alcanzado los resultados. Generalmente, se relaciona la evaluación con mecanismos para describir, medir, clasificar y certificar; pero no como una función pedagógica y que debe contemplar la comprensión, regulación y la mejora del proceso de enseñanza aprendizaje, tanto en el estudiante como en el profesor.

En el Perú y particularmente en las instituciones educativas de Castilla-Piura, se han venido dando cambios progresivos en la educación. Sin embargo estos avanzan de manera muy lenta, en muchas de éstas 
instituciones se sigue enseñando con estrategias obsoletas que no toman en cuenta al papel activo que debe tener el estudiante, según el enfoque constructivista. La evaluación solo se mira desde los resultados finales sin ver los procesos, que es el centro de atención en la educación, según el modelo constructivista. Es más, solo se utilizan estrategias denominadas de fase inicial para el aprendizaje y escasamente se utilizan las de fase intermedia y final; estas limitaciones pueden tener serias repercusiones en la evaluación de los aprendizajes, sobre todo en lo relacionado a la evaluación de los procesos.

Dentro de las estrategias de enseñanza no se considera a la evaluación como parte del proceso de aprendizaje de los estudiantes y la relación entre estos aspectos, lo que ha generado el interés para desarrollar esta investigación. Sin embargo, estas áreas de la educación se han venido estudiando por separado y bajo distintos parámetros. Por lo cual resulta necesario estudiar las posibles relaciones entre ambas en instituciones educativas. Es por ello, que se consideró como Objetivo General: Determinar la relación entre las estrategias de enseñanza basadas en un enfoque constructivista y la evaluación de los aprendizajes en las instituciones educativas de Castilla-Piura, en el 2015. Y como objetivos específicos:

1. Identificar el nivel de uso de las estrategias de enseñanza basadas en un enfoque constructivista de las Instituciones Educativas de Castilla, 2015.

2. Identificar el nivel de evaluación de los aprendizajes de las Instituciones Educativas de Castilla, 2015.

3. Establecer la relación que existe entre Las estrategias de fase inicial para el aprendizaje del enfoque constructivista y la evaluación de los aprendizajes.
4. Establecer la relación que existe Las estrategias de fase intermedia para el aprendizaje del enfoque constructivista y la evaluación de los aprendizajes.

5. Establecer la relación que existe Las estrategias de fase terminal para el aprendizaje del enfoque constructivista y la evaluación de los aprendizajes.

Dada la importancia que han adquirido tanto las estrategias de enseñanza basadas en el enfoque constructivista y la evaluación de los aprendizajes, la presente investigación se justifica por varias razones expuestas a continuación: En lo teórico, si bien existen teorías que fundamentan cada uno de estos aspectos, no hay estudios empíricos que involucren a los dos y que indiquen cómo uno puede determinar en el otro; por lo tanto, el estudio pretende brindar un aporte en este sentido.

En lo práctico, el estudio se justifica porque beneficiará a la comunidad educativa; el conocimiento de la relación entre las dos áreas investigadas permitirá emprender acciones directas sobre ellas para que la enseñanza y el aprendizaje en las escuelas sea óptimo. Asimismo, la investigación tiene relevancia social porque permite identificar cómo los docentes de las instituciones investigadas utilizan las estrategias de enseñanza y si estas están basadas en el enfoque constructivista; también permite caracterizar la evaluación del aprendizaje y de qué manera éstas, son influenciadas por las estrategias que utilizan los docentes.

La presente investigación quiere dar a conocer el enfoque pedagógico Constructivista, el cual está basado en la observación y el estudio científico, de cómo el niño aprende. Según este enfoque el ser humano construye sus conocimientos y aprendizajes a través de la percepción que tienen del mundo que los 
rodea y de sus propias experiencias en su interacción con él. El estudiante al percibir el mundo externo, lo asimila y lo incorpora a sus conocimientos previos, incorporándola o rechazándola, según lo indica Ausubel (1976).

En tal sentido, identificó como antecedentes a Martínez y Zea (2004), en su estudio descriptivo sobre Estrategias de enseñanza basadas en un enfoque constructivista (Universidad de Carabobo, Venezuela), concluye que hay sólidos fundamentos teóricos para orientar el enfoque constructivista hacia el proceso de enseñanza aprendizaje y para implementar acciones que permitan que el docente pueda desarrollar sus funciones en el aula. Las estrategias propuestas por estos autores, bajo este enfoque, indican que a través de ellas los alumnos podrán reconstruir sus ideas, conocimientos y experiencias de aprendizaje dentro del ambiente que los rodea. En otras palabras, estas acciones le permitirán al estudiante "construir" su conocimiento a partir de la realidad y de la interacción con otras personas; el alumno es el responsable final de su aprendizaje, el construye el conocimiento por sí mismo, relacionando la información acumulada de sus experiencias con la nueva y dándole significado. Pero es fundamental el rol del docente constructivista y el apoyo del entorno en el que se desenvuelve. El papel preponderante el docente para fomentar la generación de conocimientos a partir de las experiencias y necesidades y para crear un ambiente en donde los estudiantes se puedan expresar libremente, sin temor a equivocarse es muy importante. En tal sentido, esta investigación ha aporta conocimientos sobre la importancia de la práctica docente basada en estrategias con enfoque constructivista, para el desarrollo del estudiante.

Por otra parte, la investigación de Castro, Peley y Morillo (2006) sobre "La práctica pedagógica y el desarrollo de estrategias instruccionales desde el enfoque constructivista" (Universidad del Zulia, Venezuela), tuvo como fundamento un conjunto de elementos conceptuales y de estrategia que el docente puede aplicar en el aula como parte de su práctica pedagógica. Los autores concluyen que desde la concepción constructivista, el docente juega un rol importante no solo para transmitir conocimientos, para guiar y servir como facilitador del aprendizaje, sino también como mediador de los mismos. Por lo tanto el estudio concluye que la finalidad de la educación en las instituciones educativas es fomentar el crecimiento personal del alumno, en el contexto en el que se desenvuelve. De esta manera, la labor de los docentes permite descubrir que sucede con los estudiantes en cada etapa de su vida. Conociendo sus acciones, su forma de pensar, su desarrollo, sus capacidades y limitaciones, el docente estará en mayor capacidad para ayudarlos, acompañarlos y estimularlos de la mejor manera, como propone el enfoque constructivista.

Por otra parte, en cuanto a la Evaluación de Aprendizajes la presente investigación se apoyó en el estudio de Araujo (2010) sobre la función pedagógica de la evaluación de una perspectiva constructivista (Universidad de Nariño, Colombia), la cual buscaba proyectar algunas perspectivas conceptuales y epistemológicas que contribuyen a orientar y promover la función pedagógica de la evaluación del aprendizaje significativo del inglés como lengua extranjera. La investigación destaca la función pedagógica en la evaluación y considera que debe contemplar la comprensión, regulación y la mejora del proceso de enseñanza aprendizaje, tanto en el estudiante como en el profesor. Esto resulta importante porque proporciona elementos de análisis donde se evidencia que la evaluación abarca obviamente los productos, pero también los procesos y los contextos de 
aprendizaje permitiendo reorientarlos cuando se requiera para acomodarlo a las necesidades e intereses de los estudiantes, a su ritmo de desarrollo y aprendizaje y a las características del contexto. La evaluación de aprendizajes permite formar estudiantes pensadores, críticos, que sean capaces de construir sus propios procesos de aprendizaje, lo cual responde a los planteamientos del enfoque constructivista.

La perspectiva teórica de la investigación se sustenta en la relación de las dos variables: (1) Estrategias de enseñanza basadas en un enfoque constructivista y (2) Evaluación de los aprendizajes. En la primera, se tiene como centro el constructivismo, corriente epistemológica preocupada por identificar los problemas que enfrenta la formación del conocimiento del ser humano. Delval (1997), encontró algunos elementos del constructivismo en el pensamiento de Vico, Kant, Marx y Darwin; estos autores ya planteaban en forma similar a los constructivistas de hoy que "los seres humanos son producto de su capacidad para adquirir conocimientos y para reflexionar sobre sí mismos" (p.257) lo que ha facilitado predecir, explicar y dominar la naturaleza y construir su propia cultura. Se desprende de estas ideas que los sujetos construyen sus conocimientos en forma activa; éstos no llegan de manera pasiva del ambiente.

Para Porlán (1995), en el constructivismo "el conocimiento se adquiere por un proceso de construcción, o mejor, de auto-construcción y no acumulación de información proveniente del exterior" (p.6o). Por lo tanto, la escuela debe garantizar por intermedio de sus profesores, que la enseñanza conduzca a un aprendizaje significativo y que estimule al estudiante a participar, a indagar, a generarse interrogantes y a responder de acuerdo a su propia reflexión, capacidad y experiencia.
Según esta teoría, el sujeto debe aprender de sus errores para aproximarse a la verdad.

Lo anterior deja en claro cuál es el papel que juega el educador; según el enfoque del constructivismo, el profesor no es un simple transmisor de conocimientos, su función se extiende más allá. Se trata de crear las condiciones adecuadas para que el alumno sea capaz de interactuar con el profesor mismo y con sus compañeros, así como con el material y las situaciones a las que se enfrenta; hasta finalmente concretar la construcción personal. Para ello, el docente debe tener una sólida formación pedagógica, que le permita orientar, facilitar y actuar como mediador, de las necesidades del alumno, que le permita lograr su desarrollo y su incorporación y desenvolvimiento en cualquier medio. El resultado de estas acciones, en conjunto con una interacción constante con la realidad, es que los alumnos estarán en capacidad de seleccionar, organizar y transformar la información, atribuyéndoles un significado, es decir, construyen un aprendizaje significativo. Para Ausubel (1976)

El aprendizaje y la retención de carácter significativo, basados en la recepción, son importantes en la educación porque son los mecanismos humanos «par excellence» para adquirir y almacenar la inmensa cantidad de ideas y de información que constituye cualquier campo de conocimiento. Sin duda la adquisición y la retención de grandes corpus de información es un fenómeno impresionante si tenemos presente, en primer lugar, que los seres humanos, a diferencia de los ordenadores, sólo podemos captar y recordar de inmediato unos cuantos elementos discretos de información que se presenten una sola vez y, en segundo lugar, que la memoria para 
listas aprendidas de una manera memorista que son objeto de múltiples presentaciones es notoriamente limitada tanto en el tiempo como en relación con la longitud de la lista, a menos que se sometan a un intenso sobre aprendizaje $y$ a una frecuente reproducción. La enorme eficacia del aprendizaje significativo se basa en sus dos características principales: su carácter no arbitrario y su sustancialidad (no literalidad) (p.47)

De acuerdo a este autor, los estudiantes no comienzan su aprendizaje de cero, ellos contribuyen al proceso de aprendizaje con sus experiencias y conocimientos, de tal manera que éstos influyen en los demás estudiantes. Esto puede mejorar el proceso de aprendizaje y para hacerlo significativo. Por lo cual, el aprendizaje significativo se asume como el proceso donde se relaciona un nuevo conocimiento o una nueva información con la estructura cognitiva de la persona que aprende de forma no arbitraria y no literal.

Por ello, el aprendizaje significativo es dinámico y progresivo y ocurre en una serie de fases; Shuell (1990) recoge los aportes desde diferentes enfoques cognitivos y tres fases para este tipo de aprendizaje, las cuales están relacionadas al enfoque constructivista y fueron trabajadas en la presente investigación. Estas fases son: estrategias de fase inicial, estrategias de fase intermedia y estrategias de fase final para el aprendizaje.

Con respecto a la fase inicial, Shuell (citado por Díaz-Barriga y Hernández, 2002) describe los siguientes aspectos:

- El estudiante percibe la información como constituida por piezas o partes aisladas conceptualmente.
- El estudiante tiende a memorizar o interpretar de acuerdo a sus posibilidades estas piezas y para ello usa conocimientos ya conocidos (Conocimiento esquemático).

- El procedimiento de la información es global y éste se basa en: escaso conocimiento específico del campo de estudio, estrategias generales independientes de tema, uso de conocimientos de otro tema para interpretar la información (para comparar y usar analogías).

- La información aprendida es concreta y vinculada al contexto específico.

- Uso predominante de estrategias de repaso para aprender la información.

- Gradualmente el estudiante va construyendo un panorama global del campo de estudio o del material que va a aprender, para lo cual usa su conocimiento esquemático, establece analogías (con otras áreas que conoce mejor) para representarse ese nuevo campo de estudio, construye suposiciones basadas en experiencias previas, etc.

Con respecto a la fase intermedia, Shuell (citado por Díaz-Barriga y Hernández, 2002) describe los siguientes aspectos:

- El estudiante empieza a encontrar relaciones y similitudes entre las partes aisladas. Llega a configurar esquemas y mapas cognitivos acerca del material y manejo de contenidos en forma progresiva. Sin embargo, estos esquemas no permiten aún que el aprendiz se conduzca en forma automática o autónoma.

- Comprensión más profunda de los contenidos al ser aplicarlos a situaciones diversas.

- Hay más oportunidad para reflexionar sobre la situación, material y contenidos. 
- El conocimiento llega a ser más abstracto, es decir, menos dependiente del contexto donde originalmente fue adquirido.

- Es posible el empleo de estrategias más sofisticadas tales como: mapas conceptuales y redes semánticas (para realizar conductas metacognitivas), así como para usar la información en la solución de tareasproblema, donde se requiera la información a aprender.

Como se puede observar en la fase intermedia, los estudiantes empiezan a integrar las partes aisladas, encontrando relaciones y similitudes entre ellas; pero aún no actúan de forma autónoma. El conocimiento adquirido se vuelve aplicable a otros contextos y los docentes pueden crear estrategias más complejas y mejor elaboradas; los estudiantes adquieren la capacidad de solucionar tareas y problemas con la información aprendida.

Con respecto a la fase final, Shuell (citado por Barriga y Hernández, 2005) describe los siguientes aspectos:

- Los conocimientos que comenzaron a ser elaborados en esquemas o mapas cognitivos en la fase anterior, llegan a estar más integrados $\mathrm{y}$ a funcionar con mayor autonomía.

- Como consecuencia de ello, las actividades comienzan a ser más automáticas y a exigir un menor control consciente. La ejecución llega a ser automática y sin tanto esfuerzo.

- El aprendizaje durante esta fase probablemente consiste en: a) la acumulación de información a los esquemas preexistentes y b) aparición progresiva de interrelaciones de alto nivel en los esquemas.

En la tercera fase o fase final, Nieves (2012) indica que los conocimientos logran mayor integración y funcionan con mayor autonomía; los sujetos adquieren la capacidad para la realización de tareas, como la solución de problemas, respuestas a diferentes interrogantes, etc.

En relación a la variable Evaluación de los aprendizajes, Bernard (2000) señala que es prácticamente imposible hablar de evaluación sin plantearse la delimitación de lo que se entiende por aprendizaje. En este sentido, la evaluación está estrechamente ligada al aprendizaje, al uso de estrategias y los modelos educativos al momento de la enseñanza. Partiendo de esta premisa, se identifican diferentes criterios para la evaluación, los cuales están ligados a las estrategias y modelos utilizados.

No obstante, esta concepción de la evaluación es muy compleja y no hay acuerdo sobre ella. Existen algunos autores que la definen en forma similar a Bernard y que sirven de base al presente estudio. Tales como señala María Alfaro (citado por Herrera, 2009) valuar es "Comparar lo deseado con lo realizado" (s.n.), mientras que Forns (citado por Herrera, 2009) la define como el proceso de "Estimar cuantitativamente y cualitativamente el valor, la importancia o la incidencia de determinados objetos, personas o hechos" (s.n.).

Por otra parte, Lafourcade (citado por Herrera, 2009) indica que la evaluación es el
Medio que permite observar y describir con mayor precisión los aspectos cuantitativos $\mathrm{y}$ cualitativos de la estructura, el proceso y el producto de la educación. Su finalidad es facilitar una predicción y un control lo más exacto posible del proceso educativo. (s.n.)

Este autor también considera a la evaluación, como "una etapa del proceso educacional que tiene por fin comprobar de 
modo sistemático en qué medida se han logrado los resultados previstos en los objetivos que se hubieran especificado con antelación" (s.n.)

Estas son algunas de las definiciones, dentro de las múltiples que se encontraron durante la revisión bibliográfica, sin embargo en todas ellas tienen algo en común, la evaluación es una actividad que permite no solo valorar, sino también investigar los resultados alcanzados por los alumnos y permite adecuar permanentemente el sistema educativo a las demandas sociales y a las necesidades de los estudiantes.

El presente estudio está centrado en la evaluación de los aprendizajes, esta actividad no se circunscribe solo a comprobar resultados del aprendizaje, como el rendimiento académico; esta cumple múltiples funciones, como la de servir como base para definir los significados pedagógicos y sociales, cumple la función social, cumple la función de control, la función pedagógica entre las más importantes. En la investigación se ha considerado a la función social, función de control, función pedagógica y función de control, como dimensiones de la evaluación de los aprendizajes.

En cuanto a la función social, González y Pérez (2000) indica que son las que tienen que ver con la certificación del saber, la acreditación, la selección, la promoción. En función a los resultados de esta función, las instituciones educativas certifican la posesión del saber y la competencia y entregan títulos. Sin embargo se corre el riesgo que las personas que lo poseen no necesariamente sean competentes; los títulos garantizan formalmente el saber, pero como dice (Bourdieu y Passerson, 1981), no asegurar que esa garantía tenga una certidumbre total. Existen otros casos en los cuales la persona es competente para las tareas que desempeña, pero no tienen el título que lo acredite.

En segundo lugar, se encuentra la función de control, la cual se orienta a ejercer el poder de control a los evaluadores y puede tener implicancias en la vida de los vida de los educandos; según González y Pérez (200o) este tipo de evaluación es un instrumento potente para ejercer el poder y la autoridad de unos sobre otros, del evaluador sobre los evaluados. En el ámbito educativo le da poder al profesor, le confiere capacidad de tomar decisiones en cuanto a los aspectos que son o no relevante.

La tercera función, se refiere a la función pedagógica, la cual se encuentra dentro de las funciones constructivistas de la evaluación. González (2000) indica que cumple con el papel orientador, de diagnóstico, de pronóstico, es creadora de un buen clima escolar, sirve de afianzamiento del aprendizaje, así como para la individualización, para la retroalimentación, motivación y de preparación de los estudiantes para la vida.

\section{MÉTODO}

Dada la naturaleza del estudio y con el fin de cumplir con los objetivos e hipótesis de investigación, se ha utilizado el enfoque cuantitativo, siendo un estudio de carácter no experimental de tipo descriptivo-correlacional. En primer lugar se estableció la operacionalización de las dos variables: (1) Estrategias de enseñanza basadas en un enfoque constructivista y (2) Evaluación de los aprendizajes. La muestra está compuesta por 88 docentes de cuatro Instituciones educativas de Castilla-Piura, que fueron el total de la población. Dado que es una población pequeña es posible estudiar a todos sus elementos, no se ha utilizado ningún tipo de muestreo.

Para la recolección de datos, se utilizó la técnica de la encuesta y como instrumento dos cuestionarios que tienen la finalidad de recoger información sobre cada una de las variables. Sus preguntas responden a los indicadores de cada una de las variables de investigación. Dichos instrumentos fueron validados según el 
criterio de "Juicio de expertos" y evaluada su confiabilidad utilizando el procedimiento Alfa de Cronbahc; el cual evalúa la consistencia y coherencia interna de las preguntas. Los valores obtenidos fueron 0.896 , para el cuestionario de estrategias de enseñanza y de 0.738 para el de evaluación de aprendizajes; ambos califican dentro del rango de confiabilidad alto, lo que permitió aplicar el cuestionario con la confianza de cumplir con los objetivos de la investigación.

Una vez que se recogió la información mediante la aplicación de los instrumentos de recolección de datos, se creó una base de datos en el programa estadístico IBM SPSS 22.0; en este programa se elaboraron tablas en las que se presentan los resultados con frecuencias absolutas y porcentuales. El programa también permitió contrastar las hipótesis de investigación a través del coeficiente de correlación de Spearman. Este indicador estadístico se usa porque los constructos o variables provienen de preguntas medidas en escala ordinal.

\section{RESULTADOS Y DISCUSIÓN}

En esta sección se presentan los resultados de la investigación logrados tras la aplicación de dos instrumentos de recolección de datos. Las respuestas de cada instrumento están estructuradas en escalas de Likert, con cinco alternativas: Nunca, Casi nunca, A veces, Casi siempre y Siempre, a las que se les asignó valores del 1 al 5. Para evaluar el nivel alcanzado en cada una de las dimensiones de las variables se promedió las preguntas que están incluidas en cada una de las respuestas y para la variable en general se siguió el mismo procedimiento, es decir se calculó el promedio de todas las preguntas del cuestionario. Si el promedio se acercó al valor 1 se le asignó el nivel muy deficiente, si el promedio se aproxima a los 2 puntos se le asignó el nivel deficiente, a un promedio de 3 puntos le corresponde el nivel regular, a un promedio de 4 puntos el nivel bueno y a un promedio de 5 puntos el nivel muy bueno.

A continuación, se presentan los apartados medidos, teniendo en cuenta los objetivos de la investigación.

1.- Evaluación de las estrategias de enseñanza basadas en un enfoque constructivista de las Instituciones Educativas de Castilla, 2015. De acuerdo a los resultados el $51.9 \%$ de los docentes evidencian un nivel bueno en el uso de las estrategias de fase inicial. Mientras las estrategias de fase intermedia son usadas por una mayoría de $61.7 \%$ en forma regular; en el caso de las estrategias de fase terminal los resultados dejan en evidencia que son utilizadas en forma regular por un $48.1 \%$ y deficiente por un $46.9 \%$ de los docentes. Estos resultados dan como consecuencia que las estrategias de enseñanza son utilizadas en un $61.7 \%$, lo cual las ubica en el nivel de uso regular.

Estos hallazgos indican que los docentes mayormente utilizan las estrategias de fase inicial, es decir, emplean el conocimiento esquemático, procedimientos de información global. Muestran un buen conocimiento sobre el esquema preexistente a aprender y generalmente utilizan estrategias independientes del domino. El estudio también deja en claro que los docentes utilizan conocimientos de otros esquemas para interpretar la información, sobre todo para comparar y usar analogías. La información aprendida por los docentes es concreta y está vinculada a un contexto específico; los resultados también dejan en claro que los docentes utilizan estrategias de repaso para aprender la información.

Con respecto a las estrategias de fase intermedia los resultados indican que los docenes no tienen un panorama global del 
dominio y material que utilizan para los aprendizajes y para establecer analogías con otros dominios que conocen mejor, para representar ese nuevo dominio. Las estrategias de fase final también son las que menos se utilizan; es decir, los docentes no suelen construir suposiciones basadas en experiencias previas, tampoco utilizan muchos mapas cognitivos o estrategias de dominio para la realización de tareas, solución de problemas y para dar respuestas a preguntas técnicas. Todos estos datos conducen a una valoración regular de las estrategias de enseñanza, correspondiente al $61.7 \%$; sólo el $9.9 \%$, las considera buenas y el $28.4 \%$, deficientes.

2.- Evaluación de los aprendizajes de los estudiantes de las Instituciones Educativas de Castilla, 2015. En cuanto a la evaluación de los aprendizajes, el estudio da cuenta que la gran mayoría de docentes evidencia un buen nivel en el uso de la función social con $66.7 \%$, y la función de control con un $61.7 \%$. Mientras que en la función pedagógica predomina niveles deficientes con un 38.3\%.

En cuanto a la función social, este resultado implica que los docentes en sus actividades de evaluación certifican los saberes, los acreditan, realizan correctamente la función de selección y de promoción. En lo referente a la función de control, es claro que se observa y analiza exhaustivamente la realidad educativa y ponen en un contexto social los resultados de la evaluación, por sus implicancias en la vida de los educandos. En este caso también es importante señalar, que hay un porcentaje significativo de $14.8 \%$, que no logran desarrollar adecuadamente ninguna de estas dos funciones.

La función pedagógica es la que no logra una percepción muy favorable; pues está por debajo de las otras dimensiones. En este caso, los docentes consideran que no siempre cumplen con la función orientadora, de diagnóstico y pronóstico. Las actividades pedagógicas tampoco crean un buen ambiente escolar, afianzando y personalizando los aprendizajes, utilizando para ello, la retroalimentación, la motivación. Todas estas acciones, que deberían orientar y preparar a los estudiantes para que se desenvuelvan en su vida cotidiana, no se están logrando a cabalidad. En este caso sólo el 19\% de los investigados, cumplen bastante bien con dicha función. Los resultados generales muestran una evaluación de aprendizajes que se puede catalogar como regular con un $56,8 \%$ y un $33.3 \%$ que la realiza bien.

3.- Relación entre las estrategias de fase inicial y la evaluación de los aprendizajes de las Instituciones Educativas de Castilla, 2015. Los resultados anteriores indican que la mayoría de docenes que utilizan las estrategias de fase inicial en forma deficiente, también evalúan los aprendizajes de manera deficiente. En cambio, los que realizan una buena evaluación de los aprendizajes, son aquellos docentes que utilizan bien las estrategias de fase inicial. En este caso hay una tendencia clara que la evaluación de aprendizajes mejora, en tanto mejora la utilización de las estrategias de fase inicial.

Contrastación de la hipótesis específica 1: Existe relación significativa entre las estrategias de fase inicial y la evaluación de los aprendizajes de las Instituciones Educativas de Castilla, 2015. El análisis de correlación confirma que hay una relación significativa (Sig.<0.05) entre las estrategias de fase inicial y la evaluación de los aprendizajes.

Relación entre las estrategias de fase intermedia y la evaluación de los aprendizajes de las Instituciones Educativas de Castilla, 2015. De acuerdo a los resultados, los docentes que utilizan las estrategias de fase intermedia en forma deficiente, en su mayoría, realizan una evaluación regular de los aprendizajes. Sin 
embargo, los docentes que utilizan bien dichas estrategias, mayormente realizan regular o bien la evaluación de los aprendizajes. En este caso también hay una tendencia a mejorar la evaluación de los aprendizajes cuando se utilizan mejor las estrategias de fase intermedia.

Contrastación de la hipótesis específica 2: Existe relación significativa entre las estrategias de fase intermedia y la evaluación de los aprendizajes de las Instituciones Educativas de Castilla, 2015. Los resultados indican que no hay una correlación significativa (Sig.>0.05) entre las estrategias de fase inicial y la evaluación de los aprendizajes; este resultado no permite aceptar la hipótesis de investigación formulada.

Relación entre las estrategias de fase terminal y la evaluación de los aprendizajes de las Instituciones Educativas de Castilla, 2015. Al relacionar las estrategias de fase terminal con la evaluación de los aprendizajes, los resultados indican que ésta es mayormente regular, independientemente de la forma como los docentes utilizan las estrategias de fase intermedia. Los resultados también indican que la evaluación de aprendizajes buena se refleja mayormente en los docentes que utilizan las estrategias en forma regular. En este caso no hay una tendencia clara que la evaluación de aprendizajes sea mejor cuando se utiliza bien las estrategias de fase terminal.

Contrastación de la hipótesis específica 3: Existe relación significativa entre las estrategias de fase terminal y la evaluación de los aprendizajes de las Instituciones Educativas de Castilla, 2015. Los resultados de la prueba indican que las estrategias de fase terminal no se relacionan de manera significativa (Sig.>0.05) con la evaluación de los aprendizajes; este resultado no permite aceptar la hipótesis de investigación planteada.
Relación que existe entre las estrategias de enseñanza basadas en un enfoque constructivista y la evaluación de los aprendizajes de las Instituciones Educativas de Castilla, 2015. El estudio deja en claro que la evaluación de los aprendizajes regular se presenta básicamente en los docentes que utilizan las estrategias de enseñanza en forma regular o buena. En cambio, el nivel bueno en dichas estrategias se presenta con mayor frecuencia en los docentes que utilizan bien las estrategias de enseñanza. En este caso se observa que la evaluación buena de los aprendizajes es mucho mejor en los docentes que utilizan muy bien dichas estrategias.

Contrastación de la hipótesis general: Existe relación significativa entre las estrategias de enseñanza basadas en el enfoque constructivista y la evaluación de los aprendizajes de las Instituciones Educativas de Castilla, 2015. El estudio deja en claro que la evaluación de aprendizajes se relaciona de manera significativa (Sig.<0.05) con las estrategias de enseñanza, lo que conduce a aceptar la hipótesis de investigación planteada.

El objetivo principal del estudio es evaluar la relación que existe entre las estrategias de enseñanza basadas en el enfoque constructivista y la evaluación de los aprendizajes de las instituciones Educativas de Castilla-Piura, 2015. Para Coll (1990), las estrategias de enseñanza desde el enfoque constructivista, vienen a ser la participación del alumno en las actividades intencionales, planificadas y sistemáticas en todas las fases del aprendizaje; por su parte, sobre la evaluación de los aprendizajes, Bernard (200o) indica que es un proceso por medio del cual los profesores buscan formarse un juicio de valor a partir de información procedente de diferentes fuentes, sobre alguna característica particular del alumno. 
Con respecto al primer objetivo de la investigación, la evaluación de las estrategias de enseñanza de las Instituciones Educativas de Castilla-Piura, el estudio deja en claro que la mayoría de estudiantes consideran que las estrategias de enseñanza, de fase inicial son bien utilizadas por parte de los docentes (51.9\%) y los docentes utilizan los esquemas vinculados a la realidad, analogías, estrategias de repaso para aprender de la información. En cuanto a las estrategias de fase intermedia los resultados indican que la mayoría de los docentes, las utilizan solo en forma regular (61.7\%); lo que indica que no siempre utilizan conocimientos del dominio de estudio, como de otros dominios que permitan afianzar el aprendizaje del estudiante; no hay un buen uso de los dominios y de los materiales para nuevos dominios, sobre todo basados en experiencias previas. Con respecto a las estrategias de fase final, los docenes tampoco las utilizan mucho, el $48.1 \%$ las usa de manera deficiente. Este resultado indica que no se utilizan mucho los mapas cognitivos. Es importante señalar los docentes mayormente utilizan las estrategias más fáciles de llevar a la práctica, fase inicial, y las menos usadas son de la fase final. Estos resultados significan además que los docentes no comprenden mucho el proceso de construcción de conocimientos de los estudiantes, lo que no les permite construir los saberes a partir de la interacción de los conocimientos previos, de las experiencias y de las necesidades.

El segundo objetivo está orientado a evaluar el nivel alcanzado en la evaluación de los aprendizajes y en general es regular $(56.8 \%)$, producto de un nivel bueno en lo concerniente a la función social $(66.7 \%)$ y función de control $(61.7 \%)$ y de un nivel entre deficiente $(38.3 \%)$ y regular $(38.3 \%)$ en la función pedagógica. Según estos resultados, los docentes acreditan los saberes y los seleccionan y promocionan a los estudiantes en función a ellos; los resultados también dejan entrever que los docentes ejercen bastante bien la función de control, observan y analizan minuciosamente la realidad educativa y las evaluaciones los ubican en el contexto social de los estudiantes. Por el contrario, la función pedagógica es la que menos avances presenta, al evidenciar un nivel regular o deficiente en la mayoría de docentes; este resultado significa que los docentes tienen dificultades para orientar a los estudiantes, y no siempre realizan un buen diagnóstico y pronóstico de los saberes; no logran crear un buen ambiente escolar, no personalizan los aprendizajes, ni motivan a los estudiantes.

El tercer objetivo relaciona las estrategias de fase inicial con la evaluación de los aprendizajes; los resultados indican que ambos aspectos se encuentran significativamente relacionados, lo cual implica que una mejora en las estrategias de fase inicial y la evaluación de los aprendizajes. Los hallazgos dejan en claro que el uso de estrategias como el conocimiento esquematizado, el uso de estrategias no solo relacionadas al tema, sino sobre otros dominios que ayuden a interpretar la información, tiene un efecto positivo sobre los aprendizajes. Los que se ven reforzados por el conocimiento de los docentes sobre el contexto específico y el uso del repaso, como una manera de reforzar el conocimiento de la información aprendida anteriormente. Teniendo en cuenta lo que dice Shuell (1990), que el aprendizaje ocurre en una serie de fases, y es precisamente en esta fase en la cual el estudiante forma su base. El autor también destaca la importancia del uso del repaso para recuperar la información suministrada con anterioridad o para entenderla mejor.

El cuatro objetivo está orientado a determinar la relación entre las estrategias de fase intermedia con la evaluación de los 
aprendizajes; el estudio muestra que ambos aspectos no se encuentran significativamente relacionados. Díaz-Barriga y Hernández (2002) indican que en esta fase el aprendiz comienza a encontrar relaciones y similitudes entre partes aisladas y llega a configurar esquemas y mapas cognitivos acerca del material y el dominio de aprendizaje. Lo que no se observa en la investigación; pues los docentes no utilizan mucho este tipo de estrategias; esto puede ser una causa de la no correlación con la evaluación de los aprendizajes. Este resultado implica además que la mayoría de docentes de las instituciones educativas investigadas, no han logrado que los estudiantes sean capaces de elaborar esquemas y mapas cognitivos acerca del material y el dominio de aprendizaje en forma progresiva. Igualmente, no los han ayudado a enlazar de manera coherente de las estrategias de esta fase con las estrategias de fase inicial.

El quinto objetivo evalúa la relación entre las estrategias de fase terminal y la evaluación de los aprendizajes; los resultados dejan en evidencia que ambos aspectos no se relacionan de manera significativa. Díaz-Barriga y Hernández (2002) señalan que en esta fase los conocimientos son más integrados y autónomos; en este caso los estudiantes actúan de manera más automática. Aquí la actuación del sujeto se basa en estrategias del dominio para la realización de sus tareas, tales como la solución de problemas y respuesta a preguntas. En el estudio, estas estrategias no son bien utilizadas, situación que no tiene mucho que ver con la evaluación de los aprendizajes, puesto que no están correlacionados. Este resultado deja en claro que aún no se ha producido la transición entre las fases; ésta, según el autor, deben ser gradual más que inmediata.

\section{CONCLUSIONES}

1. Las estrategias de enseñanza basadas en el enfoque constructivista mayormente son utilizadas en un nivel medio o regular por la mayoría de docentes de las Instituciones Educativas de Castilla; este nivel es el producto del buen uso de las estrategias de fase inicial, de un uso regular de las estrategias de fase intermedia y de un uso entre deficiente y regular de las estrategias de fase terminal.

2. El nivel de la evaluación de los aprendizajes es regular, caracterizado por una buena evaluación de la función social y de control; así como una evaluación entre regular y deficiente de la función pedagógica.

3. Las estrategias de fase inicial se relacionan de manera significativa con la evaluación de los aprendizajes. Por ello, un mejor uso de este tipo de estrategias tiene un efecto positivo en el aprendizaje de los estudiantes.

4. Las estrategias de fase intermedia no tienen un efecto significativo sobre los aprendizajes.

5. Las estrategias de fase final, no se relacionan de manera significativa con la evaluación de los aprendizajes.

6. Las estrategias de aprendizaje se relacionan de manera significativa con la evaluación de los aprendizajes; Esto implica que en la medida que se mejore el uso de dichas estrategias, se podrá mejorar más la evaluación de los aprendizajes.

\section{REFERENCIAS}

Araujo (2010) La función pedagógica de la evaluación desde una perspectiva constructivista. (Tesis doctoral. Universidad de Nariño). Recuperado de http://simposioevaluacion.udenar.edu.co/ wp-content/uploads/2014/10/LA- 
FUNCIONPEDAG\%C3\%93GICA-DE-LAEVALUACI\% $\mathrm{C}_{3} \%{ }_{3} \mathrm{~N}$-DESDE-UNAPERSPECTIVACONSTRUCTIVISTA1.pdf

Ausubel, D. P. (1976). Psicología educativa. Un punto de vista cognoscitivo. México: Ed. Trillas

Bernard, J. (200o) Modelo cognitivo de evaluación educativa: Escala de Estrategias de Aprendizaje Contextualizado. Madrid: Narcea Ediciones

Bourdieu, P. y Passerson, J. (1981). La reproducción. Elementos para una teoría del sistema de enseñanza. Barcelona: Editorial Leia

Castro, E., Peley, R. y Morillo, R. (2006) La práctica pedagógica y el desarrollo de estrategias instruccionales desde el enfoque constructivista. Revista de Ciencias Sociales. 8(3). pp. 581-587. Recuperado de http://www.scielo.org.ve/pdf/rcs/vi2n3/art1 2.pdf

Coll, C. (1990). Un marco de referencia psicológico para la educación escolar. La concepción constructivista del aprendizaje y de la enseñanza". En Marchesi, Coll y Palacios (Comp). Desarrollo psicológico y educación II. Psicología de la Educación. Madrid: Alianza

Delval, J. (1997). Hoy todos son constructivistas. Caracas: Cuaderno de Pedagogía

Díaz-Barriga, F. y Hernández, G. (2002). Estrategias docentes para un aprendizaje significativo. Una interpretación constructivista. (2 $2^{\mathrm{a}}$ ed). México: Mc Graw Hill Interamericana
González, M y Pérez, N. (200o) La evaluación del proceso de enseñanza-aprendizaje. Fundamentos básicos. Recuperado de https://docs.google.com/document/d/15BE AwrNbcKoUSHgGpeCSn9YsH4vpFap3UO ML3oDiMGs/preview?pref $=2 \&$ pli=1

Herrera (2009). La Evaluación De Los Procesos De Enseñanza aprendizaje. Recuperado de http://www.csicsif.es/andalucia/modules/ mod_ense/revista/pdf/Numero_16/ANGEL A\%2OMARIA_HERRERA_1.pdf

Martínez, E. y Zea, E. (2004). Estrategias de enseñanza basadas en un enfoque constructivista. (Tesis doctoral. Universidad de Carabobo). Recuperado de http://servicio.bc.uc.edu.ve/educacion/revi sta/a4n24/4-24-4.pdf

Nieves, E. (2012). Implementación de estrategias constructivistas en la enseñanza del álgebra, que fomenten el desarrollo de la función neurocognitivaautomonitoreo, como un estudio de caso en la sección 20 del grado octavo de la educación básica de la I. E INEM "José Félix de Restrepo. (Trabajo de Grado, Maestría. Universidad Nacional de Colombia). Recuperado de https://core.ac.uk/download/pdf/11055657. pdf

Porlán, R. (1995). Constructivismo y escuela: Hacia un modelo de enseñanza aprendizaje basado en la escuela. Sevilla: Diada Editores.

Shuell, T.J. (1990) Phases of meaningful learning. Review of Educational Research 